Journal of Education and Vocational Research

Vol. 3, No. 5, pp. 165-166, May 2012 (ISSN 2221-2590)

\title{
Between Winnicot and Lacan: A Clinical Engagement
}

Book Review

\author{
Author: Lewis A. Kirshner \\ Reviewed by: Shiva Kumar Srinivasan \\ IIPM, Chennai, India \\ shiva.srinivasan@iipm.edu
}

What does it mean to theorize in the space 'between Winnicot and Lacan'? Why would theorists of either a Winnicottian or Lacanian orientation want to occupy this space? Is the notion of 'between' the same as the phrase 'in between'? These then are some of the questions that spring immediately to mind on the status of being in between theoretical orientations in clinical psychoanalysis. An interesting point of entry into the locus of the in-between pertains to the length of the analytic hour which follows the regulatory norm of 50 minutes. When Jacques Lacan began to experiment with the length of the analytic hour in France, it not only became controversial but led to a rethink on the meta-psychological implications of the variable session (which was either longer than or shorter than the prescribed 50-minute analytic hour). Lacan's main justification for the introduction of the variable session as a methodological innovation was the fact that it could precipitate the disclosures of the unconscious and thereby generate clinical insights in psychoanalysis. The generation of these insights however was not specific to a particular session since the patient's understanding of the structure and function of his own symptoms would often emerge in-between sessions. Or, to put it simply, the locus of the in-between is a source of insights which this anthology of essays tries to explore in its own turn. Why exactly did insights emerge in-between sessions? The answer was that Lacan would end a session to convey the effect of a punctuation forcing the patient to wonder why a particular form of punctuation was invoked. Varying the length of a clinical session was like an editor's insistence that a writer should vary the typical length of a sentence to increase a reader's over-all level of receptivity to a text. Punctuation was also a way of getting the patient to increase his sense of involvement with the analytic process and move away from the realm of 'empty speech', which functions as a psychological defense, to the notion of 'full speech' that is animated by a greater understanding of psychodynamics. The notion of the inbetween then has a topological meaning which we must not overlook if we are to understand the significance of the title and its implications for clinical practice.

All the contributors to this anthology are either psychiatrists or psychoanalysts except for one contributor who is a professor of comparative literature. Most of the contributors are based in France or the United States. It is important to keep this in mind since Donald W. Winnicott was a British pediatrician-turnedpsychoanalyst. His clinical work was neither understood nor even well-known in these countries during his lifetime. A possible reason for this neglect is the fact that Winnicott not only began, but continued to use a pediatric (as opposed to a meta-psychological) orientation in his clinical work. This preoccupation with what will or will not work in the clinical situation gave him a pragmatic cast as opposed to the rationalist preoccupations in much of French psychoanalysis that tries to come to terms repeatedly with Cartesian dualism of mind versus body, and the implications of this analytic distinction for meta-psychology. Here is a simple instance of this difference in approach and how that relates to different forms of clinical intervention. Winnicott noticed that mothers usually blamed themselves for all the psychological difficulties experienced by their children, and 'mother-blaming' had become a huge attribution error in clinical discourse. That is probably why Winnicott counseled patients to work with the notion of a 'good enough' mother rather than expect the mother to function from the locus of omnipotence, and embody the role of a subject without a lack. So while Winnicott found Melanie Klein's attempts to invoke pre-Oedipal configurations interesting, he did not want to merely take her work forward or choose to play political games in the ongoing rivalry between Anna Freud and Melanie Klein. Instead, Winnicott felt that it made more sense to find a third way forward on what role mothers should play in raising a child. He wanted his approach to be both pragmatic and relevant from a clinical point of view. Winnicott normalized the challenges of motherhood as a set of commonsensical challenges and articulated an approach that could actually be deployed by mothers who would take the trouble to read his books. The idea that a pragmatic approach to psychoanalysis would appeal to so many 
psychotherapists in the U.S. and France would have taken Winnicott by surprise despite the personal interest evinced by Lacan in his work on the 'transitional object and transitional phenomena'. What this anthology demonstrates however is that Winnicott is now mainstream and even Lacanians who are mainly preoccupied by the problems and challenges of grafting structural linguistics to Freudian meta-psychology find it necessary to engage with his clinical contributions. Kirshner's intention in compiling this anthology is to stage a dialectical engagement between the leading psychoanalytic exponents of 'presence' and 'absence', or of the maternal and paternal positions, in the construction of human subjectivity through the matrix of the Oedipus complex.

Kirshner is a psychiatrist at the Harvard Medical School and has taken the lead in making the work of Lacan available to psychoanalysts in America. The nine essays collected here are an attempt to understand the translational challenges in the theory and practice of psychoanalysis. They argue that while there are different traditions in psychoanalysis based on whether they are anchored mainly in theories of absence or presence, a dialectical understanding of psychoanalysis requires us to invoke the maternal and paternal positions as structural loci within the matrix of human subjectivity in order to restitute what was lost when different traditions went their own way. A dialectical approach is mainly preoccupied with thinking through the implications of clinical innovations for meta-psychology as such rather than for a particular tradition of psychoanalysis; hence, the need to think across, through, and against the different traditions of psychoanalysis. These theoretical maneuvers become especially important when psychoanalysts have to help patients from foreign cultures or make clinical interventions where the diagnostic criteria are not the same. The ontological differences between clinical states are also at stake since the existence of certain clinical syndromes is not recognized across the board. And, again, the efficacy, if any, of psychoanalytic interventions is not clear since there are no transcendental criteria to determine what exactly constitutes a therapeutic intervention. The goal of anthologies like this is to increase the level of collaboration between psychoanalysts who belong to different traditions so that it becomes possible to situate what exactly constitutes an intervention. In addition to discussions on a range of clinical phenomena, the contributors to this volume also consider a range of transitional objects and phenomena which have applications in diverse contexts such as education, cultural studies, management development programs, and so on. The notion of a transitional space (as opposed to a performative space) is for instance an important innovation. Thinking through the implications of such a space is important because as organizational psychologists at INSEAD have argued, transitional spaces can be leveraged effectively to help executives re-calibrate their mind-sets in management development programs. It can also make it possible to put these spaces to work in the contexts of executive coaching, which consists essentially of thinking through existential options in a context that is sensitive to the 'formations of the unconscious' and informed of psychodynamic modalities. This follows from the Lacanian formulation that 'the unconscious is of the order of the unrealized'. Working-through in clinical, cultural, and existential contexts then is a way of coming to terms with the unrealized. Not only will this make it possible to manage organizational situations in a psychoanalytically informed manner, but help managers remain alert to the human propensity to act-out phenomena that pertain to unconscious conflicts in the psyche. This anthology of essays will be of help to a wide range of audiences that are willing to take up the challenge of thinking between the hitherto cloistered spaces of Donald W. Winnicott and Jacques Lacan. 